DESY $97-108$

ISSN $0418-9833$

June 1997

\title{
Evolution of $e p$ Fragmentation and Multiplicity Distributions in the Breit Frame
}

\author{
H1 Collaboration
}

\begin{abstract}
Low $x$ deep-inelastic ep scattering data, taken in 1994 at the H1 detector at HERA, are analysed in the Breit frame of reference. The evolution of the peak and width of the current hemisphere fragmentation function is presented as a function of $Q$ and compared with $e^{+} e^{-}$results at equivalent centre of mass energies. Differences between the average charged multiplicity and the multiplicity of $e^{+} e^{-}$annihilations at low energies are analysed. Invariant energy spectra are compared with MLLA predictions. Distributions of multiplicity are presented as functions of Bjorken- $x$ and $Q^{2}$, and KNO scaling is discussed.
\end{abstract}




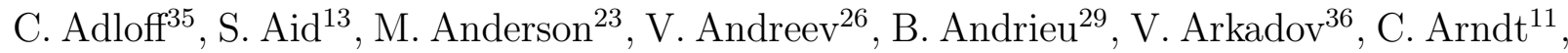
I. Ayyaz $^{30}$, A. Babaev ${ }^{25}$, J. Bähr ${ }^{36}$, J. Bán ${ }^{18}$, Y. Ban ${ }^{28}$, P. Baranov ${ }^{26}$, E. Barrelet ${ }^{30}$, R. Barschke ${ }^{11}$, W. Bartel ${ }^{11}$, U. Bassler ${ }^{30}$, H.P. Beck ${ }^{38}$, M. Beck ${ }^{14}$, H.-J. Behrend ${ }^{11}$, A. Belousov ${ }^{26}$, Ch. Berger ${ }^{1}$, G. Bernardi ${ }^{30}$, G. Bertrand-Coremans ${ }^{4}$, R. Beyer ${ }^{11}$, P. Biddulph ${ }^{23}$, P. Bispham ${ }^{23}$, J.C. Bizot $^{28}$, K. Borras ${ }^{8}$, F. Botterweck ${ }^{27}$, V. Boudry ${ }^{29}$, S. Bourov ${ }^{25}$, A. Braemer ${ }^{15}$, W. Braunschweig ${ }^{1}$, V. Brisson ${ }^{28}$, W. Brückner ${ }^{14}$, P. Bruel ${ }^{29}$, D. Bruncko ${ }^{18}$, C. Brune $^{16}$, R. Buchholz ${ }^{11}$, L. Büngener ${ }^{13}$, J. Bürger ${ }^{11}$, F.W. Büsser ${ }^{13}$, A. Buniatian ${ }^{4}$, S. Burke ${ }^{19}$, M.J. Burton ${ }^{23}$, G. Buschhorn ${ }^{27}$, D. Calvet ${ }^{24}$, A.J. Campbell ${ }^{11}$, T. Carli ${ }^{27}$, M. Charlet ${ }^{11}$, D. Clarke $^{5}$, B. Clerbaux ${ }^{4}$, S. Cocks ${ }^{20}$, J.G. Contreras ${ }^{8}$, C. Cormack ${ }^{20}$, J.A. Coughlan ${ }^{5}$, A. Courau $^{28}$, M.-C. Cousinou ${ }^{24}$, B.E. $\mathrm{Cox}^{23}$, G. Cozzika ${ }^{9}$, D.G. Cussans ${ }^{5}$, J. Cvach ${ }^{31}$,

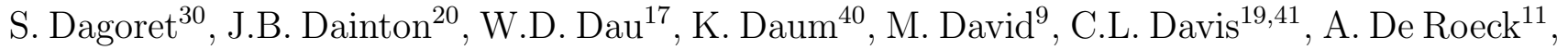
E.A. De Wolf ${ }^{4}$, B. Delcourt ${ }^{28}$, M. Dirkmann ${ }^{8}$, P. Dixon ${ }^{19}$, W. Dlugosz ${ }^{7}$, C. Dollfus ${ }^{38}$, K.T. Donovan ${ }^{21}$, J.D. Dowell ${ }^{3}$, H.B. Dreis ${ }^{2}$, A. Droutskoi ${ }^{25}$, J. Ebert ${ }^{35}$, T.R. Ebert ${ }^{20}$, G. Eckerlin ${ }^{11}$, V. Efremenko ${ }^{25}$, S. Egli ${ }^{38}$, R. Eichler ${ }^{37}$, F. Eisele ${ }^{15}$, E. Eisenhandler ${ }^{21}$, E. Elsen ${ }^{11}$, M. Erdmann ${ }^{15}$, A.B. Fahr ${ }^{13}$, L. Favart ${ }^{28}$, A. Fedotov ${ }^{25}$, R. Felst ${ }^{11}$, J. Feltesse ${ }^{9}$, J. Ferencei ${ }^{18}$, F. Ferrarotto ${ }^{33}$, K. Flamm ${ }^{11}$, M. Fleischer ${ }^{8}$, M. Flieser ${ }^{27}$, G. Flügge ${ }^{2}$, A. Fomenko ${ }^{26}$, J. Formánek ${ }^{32}$, J.M. Foster ${ }^{23}$, G. Franke ${ }^{11}$, E. Gabathuler ${ }^{20}$, K. Gabathuler ${ }^{34}$, F. Gaede ${ }^{27}$, J. Garvey ${ }^{3}$, J. Gayler ${ }^{11}$, M. Gebauer ${ }^{36}$, R. Gerhards ${ }^{11}$, A. Glazov ${ }^{36}$, L. Goerlich ${ }^{6}$, N. Gogitidze ${ }^{26}$, M. Goldberg ${ }^{30}$, D. Goldner ${ }^{8}$, K. Golec-Biernat ${ }^{6}$, B. Gonzalez-Pineiro ${ }^{30}$, I. Gorelov ${ }^{25}$, C. Grab ${ }^{37}$, H. Grässler ${ }^{2}$, T. Greenshaw ${ }^{20}$, R.K. Griffiths ${ }^{21}$, G. Grindhammer ${ }^{27}$, A. Gruber ${ }^{27}$, C. Gruber ${ }^{17}$, T. Hadig ${ }^{1}$, D. Haidt ${ }^{11}$, L. Hajduk ${ }^{6}$, T. Haller ${ }^{14}$, M. Hampel $^{1}$, W.J. Haynes ${ }^{5}$, B. Heinemann ${ }^{11}$, G. Heinzelmann ${ }^{13}$, R.C.W. Henderson ${ }^{19}$, H. Henschel ${ }^{36}$, I. Herynek ${ }^{31}$, M.F. Hess $^{27}$, K. Hewitt ${ }^{3}$, K.H. Hiller ${ }^{36}$, C.D. Hilton ${ }^{23}$, J. Hladký ${ }^{31}$, M. Höppner ${ }^{8}$, D. Hoffmann ${ }^{11}$, T. Holtom ${ }^{20}$, R. Horisberger ${ }^{34}$, V.L. Hudgson ${ }^{3}$, M. Hütte ${ }^{8}$, M. Ibbotson ${ }^{23}$, Ç. İşsever ${ }^{8}$, H. Itterbeck ${ }^{1}$, A. Jacholkowska ${ }^{28}$, C. Jacobsson ${ }^{22}$, M. Jacquet ${ }^{28}$, M. Jaffre ${ }^{28}$, J. Janoth ${ }^{16}$, D.M. Jansen ${ }^{14}$, L. Jönsson ${ }^{22}$, D.P. Johnson ${ }^{4}$, H. Jung ${ }^{22}$, P.I.P. Kalmus ${ }^{21}$, M. Kander ${ }^{11}$, D. Kant ${ }^{21}$, U. Kathage ${ }^{17}$, J. Katzy ${ }^{15}$, H.H. Kaufmann ${ }^{36}$, O. Kaufmann ${ }^{15}$, M. Kausch ${ }^{11}$, S. Kazarian ${ }^{11}$, I.R. Kenyon ${ }^{3}$, S. Kermiche ${ }^{24}$, C. Keuker ${ }^{1}$, C. Kiesling ${ }^{27}$, M. Klein ${ }^{36}$, C. Kleinwort ${ }^{11}$, G. Knies ${ }^{11}$, T. Köhler ${ }^{1}$, J.H. Köhne ${ }^{27}$, H. Kolanoski ${ }^{39}$, S.D. Kolya ${ }^{23}$, V. Korbel ${ }^{11}$, P. Kostka ${ }^{36}$, S.K. Kotelnikov ${ }^{26}$, T. Krämerkämper ${ }^{8}$, M.W. Krasny ${ }^{6,30}$, H. Krehbiel ${ }^{11}$, D. Krücker ${ }^{27}$, A. Küpper ${ }^{35}$, H. Küster ${ }^{22}$, M. Kuhlen ${ }^{27}$, T. Kurča ${ }^{36}$, J. Kurzhöfer ${ }^{8}$, B. Laforge ${ }^{9}$, M.P.J. Landon ${ }^{21}$, W. Lange ${ }^{36}$, U. Langenegger ${ }^{37}$, A. Lebedev ${ }^{26}$, F. Lehner ${ }^{11}$, V. Lemaitre ${ }^{11}$, S. Levonian ${ }^{29}$, M. Lindstroem ${ }^{22}$, F. Linsel ${ }^{11}$, J. Lipinski ${ }^{11}$, B. List ${ }^{11}$, G. Lobo ${ }^{28}$, J.W. Lomas ${ }^{23}$, G.C. $L{ }^{12}$, V. Lubimov ${ }^{25}$, D. Lüke 8,11 , L. Lytkin ${ }^{14}$, N. Magnussen ${ }^{35}$, H. MahlkeKrüger $^{11}$, E. Malinovski ${ }^{26}$, R. Maraček ${ }^{18}$, P. Marage ${ }^{4}$, J. Marks $^{15}$, R. Marshall ${ }^{23}$, J. Martens ${ }^{35}$, G. Martin ${ }^{13}$, R. Martin ${ }^{20}$, H.-U. Martyn ${ }^{1}$, J. Martyniak ${ }^{6}$, T. Mavroidis ${ }^{21}$, S.J. Maxfield ${ }^{20}$, S.J. McMahon ${ }^{20}$, A. Mehta ${ }^{5}$, K. Meier ${ }^{16}$, P. Merkel $^{11}$, F. Metlica ${ }^{14}$, A. Meyer ${ }^{11}$, A. Meyer ${ }^{13}$, H. Meyer ${ }^{35}$, J. Meyer ${ }^{11}$, P.-O. Meyer ${ }^{2}$, A. Migliori ${ }^{29}$, S. Mikocki ${ }^{6}$, D. Milstead ${ }^{20}$, J. Moeck ${ }^{27}$, F. Moreau ${ }^{29}$, J.V. Morris ${ }^{5}$, E. Mroczko ${ }^{6}$, D. Müller ${ }^{38}$, T. Walter ${ }^{38}$, K. Müller ${ }^{11}$, P. Murín ${ }^{18}$, V. Nagovizin ${ }^{25}$, R. Nahnhauer ${ }^{36}$, B. Naroska ${ }^{13}$, Th. Naumann ${ }^{36}$, I. Négri ${ }^{24}$, P.R. Newman ${ }^{3}$, D. Newton ${ }^{19}$, H.K. Nguyen ${ }^{30}$, T.C. Nicholls ${ }^{3}$, F. Niebergall ${ }^{13}$, C. Niebuhr ${ }^{11}$, Ch. Niedzballa ${ }^{1}$, H. Niggli ${ }^{37}$, G. Nowak $^{6}$, T. Nunnemann ${ }^{14}$, M. Nyberg-Werther ${ }^{22}$, H. Oberlack ${ }^{27}$, J.E. Olsson ${ }^{11}$, D. Ozerov ${ }^{25}$, P. Palmen ${ }^{2}$, E. Panaro ${ }^{11}$, A. Panitch ${ }^{4}$, C. Pascaud ${ }^{28}$, S. Passaggio ${ }^{37}$, G.D. Patel ${ }^{20}$, H. Pawletta ${ }^{2}$, E. Peppel ${ }^{36}$, E. Perez ${ }^{9}$, J.P. Phillips ${ }^{20}$, A. Pieuchot ${ }^{24}$, D. Pitzl ${ }^{37}$, R. Pöschl ${ }^{8}$, G. Pope ${ }^{7}$, B. Povh ${ }^{14}$, S. Prell ${ }^{11}$, K. Rabbertz ${ }^{1}$, P. Reimer ${ }^{31}$, H. Rick ${ }^{8}$, S. Riess $^{13}$, E. Rizvi ${ }^{21}$, 
P. Robmann ${ }^{38}$, R. Roosen ${ }^{4}$, K. Rosenbauer ${ }^{1}$, A. Rostovtsev ${ }^{30}$, F. Rouse ${ }^{7}$, C. Royon ${ }^{9}$,

K. Rüter ${ }^{27}$, S. Rusakov ${ }^{26}$, K. Rybicki ${ }^{6}$, D.P.C. Sankey ${ }^{5}$, P. Schacht ${ }^{27}$, S. Schiek ${ }^{13}$, S. Schleif ${ }^{16}$, P. Schleper ${ }^{15}$, W. von Schlippe ${ }^{21}$, D. Schmidt ${ }^{35}$, G. Schmidt ${ }^{13}$, L. Schoeffel ${ }^{9}$, A. Schöning ${ }^{11}$, V. Schröder ${ }^{11}$, E. Schuhmann ${ }^{27}$, B. Schwab ${ }^{15}$, F. Sefkow ${ }^{38}$, A. Semenov ${ }^{25}$, V. Shekelyan ${ }^{11}$, I. Sheviakov ${ }^{26}$, L.N. Shtarkov ${ }^{26}$, G. Siegmon ${ }^{17}$, U. Siewert ${ }^{17}$, Y. Sirois ${ }^{29}$, I.O. Skillicorn ${ }^{10}$, T. Sloan ${ }^{19}$, P. Smirnov ${ }^{26}$, M. Smith ${ }^{20}$, V. Solochenko ${ }^{25}$, Y. Soloviev ${ }^{26}$, A. Specka ${ }^{29}$, J. Spiekermann ${ }^{8}$, S. Spielman ${ }^{29}$, H. Spitzer ${ }^{13}$, F. Squinabol ${ }^{28}$, P. Steffen ${ }^{11}$, R. Steinberg ${ }^{2}$, J. Steinhart ${ }^{13}$, B. Stella ${ }^{33}$, A. Stellberger ${ }^{16}$, J. Stier ${ }^{11}$, J. Stiewe ${ }^{16}$, U. Stößlein ${ }^{36}$, K. Stolze ${ }^{36}$, U. Straumann ${ }^{15}$, W. Struczinski ${ }^{2}$, J.P. Sutton ${ }^{3}$, S. Tapprogge ${ }^{16}$, M. Taševskýt ${ }^{32}$, V. Tchernyshov ${ }^{25}$, S. Tchetchelnitski ${ }^{25}$, J. Theissen ${ }^{2}$, G. Thompson ${ }^{21}$, P.D. Thompson ${ }^{3}$, N. Tobien ${ }^{11}$, R. Todenhagen ${ }^{14}$, P. Truöl $^{38}$, G. Tsipolitis ${ }^{37}$, J. Turnau ${ }^{6}$, E. Tzamariudaki ${ }^{11}$, P. Uelkes $^{2}$, A. Usik ${ }^{26}$, S. Valkár ${ }^{32}$, A. Valkárováa ${ }^{32}$, C. Vallée ${ }^{24}$, P. Van $\mathrm{Esch}^{4}$, P. Van Mechelen ${ }^{4}$, D. Vandenplas ${ }^{29}$, Y. Vazdik ${ }^{26}$, P. Verrecchia ${ }^{9}$, G. Villet ${ }^{9}$, K. Wacker ${ }^{8}$, A. Wagener ${ }^{2}$, M. Wagener ${ }^{34}$, R. Wallny ${ }^{15}$, B. Waugh ${ }^{23}$, G. Weber ${ }^{13}$, M. Weber ${ }^{16}$, D. Wegener ${ }^{8}$, A. Wegner ${ }^{27}$, T. Wengler ${ }^{15}$, M. Werner ${ }^{15}$, L.R. West ${ }^{3}$, S. Wiesand ${ }^{35}$, T. Wilksen ${ }^{11}$, S. Willard ${ }^{7}$, M. Winde ${ }^{36}$, G.-G. Winter ${ }^{11}$, C. Wittek ${ }^{13}$, M. Wobisch ${ }^{2}$, H. Wollatz ${ }^{11}$, E. Wünsch ${ }^{11}$, J. Žáček ${ }^{32}$, D. Zarbock ${ }^{12}$, Z. Zhang ${ }^{28}$, A. Zhokin ${ }^{25}$, P. Zini ${ }^{30}$, F. Zomer ${ }^{28}$, J. Zsembery ${ }^{9}$, and M. zurNedden ${ }^{38}$,

${ }^{1}$ I. Physikalisches Institut der RWTH, Aachen, Germany ${ }^{a}$

${ }^{2}$ III. Physikalisches Institut der RWTH, Aachen, Germany ${ }^{a}$

${ }^{3}$ School of Physics and Space Research, University of Birmingham, Birmingham, UK ${ }^{b}$

${ }^{4}$ Inter-University Institute for High Energies ULB-VUB, Brussels; Universitaire Instelling Antwerpen, Wilrijk; Belgium ${ }^{c}$

${ }^{5}$ Rutherford Appleton Laboratory, Chilton, Didcot, UK ${ }^{b}$

${ }^{6}$ Institute for Nuclear Physics, Cracow, Poland ${ }^{d}$

${ }^{7}$ Physics Department and IIRPA, University of California, Davis, California, USA ${ }^{e}$

${ }^{8}$ Institut für Physik, Universität Dortmund, Dortmund, Germany ${ }^{a}$

${ }^{9}$ CEA, DSM/DAPNIA, CE-Saclay, Gif-sur-Yvette, France

${ }^{10}$ Department of Physics and Astronomy, University of Glasgow, Glasgow, UK ${ }^{b}$

${ }^{11}$ DESY, Hamburg, Germany ${ }^{a}$

${ }^{12}$ I. Institut für Experimentalphysik, Universität Hamburg, Hamburg, Germany ${ }^{a}$

${ }^{13}$ II. Institut für Experimentalphysik, Universität Hamburg, Hamburg, Germany ${ }^{a}$

${ }^{14}$ Max-Planck-Institut für Kernphysik, Heidelberg, Germany ${ }^{a}$

${ }^{15}$ Physikalisches Institut, Universität Heidelberg, Heidelberg, Germany ${ }^{a}$

${ }^{16}$ Institut für Hochenergiephysik, Universität Heidelberg, Heidelberg, Germany ${ }^{a}$

${ }^{17}$ Institut für Reine und Angewandte Kernphysik, Universität Kiel, Kiel, Germany ${ }^{a}$

${ }^{18}$ Institute of Experimental Physics, Slovak Academy of Sciences, Košice, Slovak Republic ${ }^{f, j}$

19 School of Physics and Chemistry, University of Lancaster, Lancaster, UK ${ }^{b}$

${ }^{20}$ Department of Physics, University of Liverpool, Liverpool, UK ${ }^{b}$

${ }^{21}$ Queen Mary and Westfield College, London, UK ${ }^{b}$

${ }^{22}$ Physics Department, University of Lund, Lund, Sweden ${ }^{g}$

${ }^{23}$ Physics Department, University of Manchester, Manchester, UK ${ }^{b}$

${ }^{24}$ CPPM, Université d'Aix-Marseille II, IN2P3-CNRS, Marseille, France

${ }^{25}$ Institute for Theoretical and Experimental Physics, Moscow, Russia

${ }^{26}$ Lebedev Physical Institute, Moscow, Russia ${ }^{f}$ 
27 Max-Planck-Institut für Physik, München, Germany ${ }^{a}$

28 LAL, Université de Paris-Sud, IN2P3-CNRS, Orsay, France

${ }^{29}$ LPNHE, Ecole Polytechnique, IN2P3-CNRS, Palaiseau, France

30 LPNHE, Universités Paris VI and VII, IN2P3-CNRS, Paris, France

${ }^{31}$ Institute of Physics, Czech Academy of Sciences, Praha, Czech Republic ${ }^{f, h}$

${ }^{32}$ Nuclear Center, Charles University, Praha, Czech Republic ${ }^{f, h}$

33 INFN Roma 1 and Dipartimento di Fisica, Università Roma 3, Roma, Italy

34 Paul Scherrer Institut, Villigen, Switzerland

${ }^{35}$ Fachbereich Physik, Bergische Universität Gesamthochschule Wuppertal, Wuppertal, Germany $^{a}$

${ }^{36}$ DESY, Institut für Hochenergiephysik, Zeuthen, Germany ${ }^{a}$

37 Institut für Teilchenphysik, ETH, Zürich, Switzerland ${ }^{i}$

38 Physik-Institut der Universität Zürich, Zürich, Switzerland ${ }^{i}$

${ }^{39}$ Institut für Physik, Humboldt-Universität, Berlin, Germany ${ }^{a}$

${ }^{40}$ Rechenzentrum, Bergische Universität Gesamthochschule Wuppertal, Wuppertal, Germany ${ }^{a}$

41 Visitor from Physics Dept. University Louisville, USA

a Supported by the Bundesministerium für Bildung, Wissenschaft, Forschung und Technologie, FRG, under contract numbers 6AC17P, 6AC47P, 6DO57I, 6HH17P, 6HH27I, 6HD17I, 6HD27I, 6KI17P, 6MP17I, and 6WT87P

${ }^{b}$ Supported by the UK Particle Physics and Astronomy Research Council, and formerly by the UK Science and Engineering Research Council

${ }^{c}$ Supported by FNRS-NFWO, IISN-IIKW

${ }^{d}$ Partially supported by the Polish State Committee for Scientific Research, grant no. 115/E-343/SPUB/P03/120/96

e Supported in part by USDOE grant DE F603 91ER40674

$f$ Supported by the Deutsche Forschungsgemeinschaft

$g$ Supported by the Swedish Natural Science Research Council

${ }^{h}$ Supported by GA ČR grant no. 202/96/0214, GA AV ČR grant no. A1010619 and GA UK grant no. 177

${ }^{i}$ Supported by the Swiss National Science Foundation

${ }^{j}$ Supported by VEGA SR grant no. 2/1325/96 


\section{Introduction}

In a previous publication [1] the $\mathrm{H} 1$ experiment analysed hadron production in $e p$ deepinelastic scattering (DIS) in the Breit frame [2, 3] of reference. We showed similarities between hadronic distributions in the 'current' hemisphere of this frame of reference and those in a given single hemisphere of an $e^{+} e^{-}$annihilation interaction. A similar analysis [4] was performed by the ZEUS experiment. The first H1 study analysed 1993 data resulting from $e^{-} p$ collisions. The 1994 run used incident positrons and this analysis has more than six times as many events despite tighter cuts on event quality. Since the $Q^{2}$ values investigated are still in general below the kinematic region where anything other than virtual photon exchange is relevant, the results of the two analyses are directly comparable.

It is the object of this analysis to study the evolution of the fragmentation function and charged hadronic multiplicity distributions. One aim is to continue the comparison with equivalent $e^{+} e^{-}$results as a test of the universality of properties of the partons found within a proton and those of the quarks produced in pairs from the vacuum. Another aim is to show sensitivity to the running of the QCD coupling constant and to leading order (LO) QCD processes from consideration of such hadronic final states.

After a description of the relevant parts of the apparatus (section 2) and a discussion of the treatment of the data (section 3), this paper describes the Breit frame of reference (section 4) and extends (section 5) the earlier analysis of the evolution of the fragmentation function with $Q^{2}$, where $q^{2}=-Q^{2}$ is the square of the four-momentum transferred from the incident lepton. The average charged multiplicity dependence on $Q$ (section 6 ) is used to study the effects of LO QCD processes. For the first time we present invariant energy spectra (section 7) from HERA and H1 multiplicity distributions (section 8) in the Breit frame.

\section{The H1 Detector}

The $\mathrm{H} 1$ detector is described in detail elsewhere [5]. Here, we give only a short description of the components more relevant to this analysis. We employ a right-handed coordinate system with the positive (forward) $z$ axis being in the direction of the incoming proton beam. Polar angles are measured from this direction.

Momentum measurements of charged particles are provided, in the central region of the apparatus, by two cylindrical and co-axial drift chambers [6] for $(r, \phi)$ measurement which have significantly fewer dead cells compared with the situation in 1993 . These detectors are supplemented by two z-chambers. In the forward (proton) direction the equivalent measurements are provided by three Radial and three Planar drift chamber modules, the Forward Track Detector [7]. All these track detectors are inside a uniform $1.15 \mathrm{~T}$ magnetic field. Track segments from all devices are combined to give efficient detection and momentum measurement with $\delta p / p^{2} \lesssim 1 \% / \mathrm{GeV}$ for most of the angular range used in this analysis, $10^{\circ}<\theta<160^{\circ}$.

In the polar angle range $4^{\circ}<\theta<153^{\circ}$ the trackers are surrounded by a fine-grained liquid argon (LAr) sampling calorimeter [8] with lead and steel absorber in the electromag- 
netic and hadronic sections respectively. The calorimeter cells measure hadronic energy flow and the energy of the scattered electron for high $Q^{2}$ events. The LAr calorimeter is complemented by a backward electromagnetic, lead-scintillator, calorimeter (BEMC [9]) covering the angular range $151^{\circ}<\theta<176^{\circ}$. Behind this there is a double-layer scintillator hodoscope which gives efficient background rejection by time of flight measurement. The data are derived from two separate samples corresponding to the scattered positron being triggered by, and detected in, either the BEMC or the LAr calorimeter. The transition is at a $Q^{2}$ of about $100 \mathrm{GeV}^{2}$ and these samples are referred to in this paper as, respectively, low or high $Q^{2}$ data. The trigger is already fully efficient at positron energies of $10 \mathrm{GeV}$.

\section{Data Selection and Corrections}

The full data sample, triggered as above, consists of some 150000 events taken at an ep centre of mass energy, $\sqrt{s}$, of $300 \mathrm{GeV}$. Event kinematic variables used in this analysis are calculated using only the scattered lepton, which gives both the best resolution in the chosen region and clear freedom from bias on the hadronic system studied. Throughout, the events are selected to have an identified scattered positron [10 with an energy $E_{e}^{\prime}>$ $14 \mathrm{GeV}$.

To reject beam associated background, we demand that there be no veto from the time of flight system and that a vertex is found for the event within $30 \mathrm{~cm}$ of the nominal vertex position. The low (high) $Q^{2}$ data referred to in the above section are selected to be within the limits $12<Q^{2}<100 \mathrm{GeV}^{2}\left(100<Q^{2}<8000 \mathrm{GeV}^{2}\right)$. The dimensionless inelasticity variable, $y=Q^{2} / x s$, is required to be in the range $0.05<y<0.6$. These conditions ensure that contamination from mis-identified photoproduction events is below the $1 \%$ level. Together these cuts remove $\sim 45 \%$ of the original sample. In addition, a cut is placed to safely exclude a further $\sim 5 \%$ of diffractive events which are not well-modelled with the DIS Monte Carlo programs used in this analysis and which have no analogue in $e^{+} e^{-}$interactions. We require that a total cluster energy of at least $0.5 \mathrm{GeV}$ should be observed in the region $4.4^{\circ}<\theta<15^{\circ}$.

Events are then selected in which a massless quark would be scattered through $10^{\circ}<$ $\theta<150^{\circ}$ using four momentum conservation with the scattered positron. This is inside the acceptance of the $\mathrm{H} 1$ track detectors and thus minimises corrections, at the cost of removing a further $\sim 24 \%$ of events.

There is a source of error arising from QED radiation which comes about because of an incorrect, typically overestimated, value of $Q^{2}$. The error in the boost leads to a miscalculation of the direction of the Breit frame axis. In most cases, this leads to an apparently empty, or at least depleted, region of phase space where the scattered quark fragments are expected. With improved statistics this has become a significant effect. To reduce the size of the necessary QED corrections, the new analysis utilises more severe cuts on the mass of the total hadronic system, $W$, of $W^{2}>4400 \mathrm{GeV}^{2}$ both using the scattered lepton variables, and separately from the hadronic system variables with the Jaquet Blondel method [11].

Following all of the selection procedures there are 20810 events in the low $Q^{2}$ sample 
and 1250 events at high $Q^{2}$.

In addition to these event selections there are also cuts made to reject badly measured tracks. Any tracks in the central chambers with transverse momentum below $150 \mathrm{MeV}$ and those in the forward track detectors with momentum below $500 \mathrm{MeV}$ are removed. We also remove tracks failing minimum requirements on the number of hits in a given chamber and the quality of the track fit. In order to have only the primary multiplicity, cuts are also made to exclude tracks not originating from the interaction vertex. There remains a small excess contribution due to the decays of short-lived strange particles.

To correct for this excess and for acceptance losses, we utilise the DJANGO6 [12] Monte Carlo event generator. This combines a LO perturbative QCD matrix element calculation and the colour dipole model of hadronisation with a calculation of QED radiative effects. Radiative effects remaining after our event selections have been corrected by comparing the results of Monte Carlo calculations with and without the inclusion of QED radiative effects. These corrections are at the $\sim 10 \%$ level. The detector response is simulated using a program based on the GEANT [13] package and the simulated events are reconstructed and selected using exactly the same analysis chain as with real data. The total bin-by-bin corrections made throughout this analysis are generally well within $\pm 20 \%$ and vary smoothly in any given distribution.

The largest individual sources of systematic error for this analysis originate in possible calibration errors of $\pm 1 \%$ for the BEMC and $\pm 3 \%$ for the LAr electromagnetic calorimeter energy scales. These directly affect the accuracy of Lorentz boosts and give rise to an uncertainty in the number of tracks of $\sim 5 \%$ at low $Q^{2}$ and $\sim 8 \%$ at high $Q^{2}$, irrespective of other kinematic selection. The corresponding systematic error in the Monte Carlo derived acceptance correction functions has been estimated to be $\sim 2 \%$ using several different generators 14, 15. Visual scans of real and simulated data have ascertained that the efficiency of the track detectors is modelled to an accuracy of better than $2 \%$ by our Monte Carlo simulations.

The full experimental details of how the corrected distributions of this analysis are obtained from the data may be found in reference [16].

\section{The Breit Frame of Reference}

The ep Breit frame is aligned with the hadronic centre of mass (HCM) but boosted along a common $z$ direction such that the incident virtual photon has zero energy, zero transverse momentum and a $z$ component of momentum $-Q$. As with the laboratory frame of reference, we choose the positive $z$ axis to be in the direction of the incoming proton. The negative $z$ direction is referred to as the 'current' hemisphere of the interaction. Our earlier Breit frame analysis [1] showed that multiplicities in the current region of the Breit frame depend on $Q$ and not on Bjorken $x\left(\sim Q^{2} / W^{2}\right)$, as opposed to the HCM where multiplicities depend on the natural scale, $W$ [17. In the naïve quark parton model (QPM) the massless incoming quark has energy $Q / 2$ and $z$ component of momentum $+Q / 2$, carrying an approximate fraction $x$ of the proton's momentum. After scattering it still has energy $Q / 2$, with momentum $-Q / 2$. By comparison, we thus take the equivalent to the $e^{+} e^{-}$centre of mass energy, $E^{*}$, to be $Q$. 
As compared with the HCM the Breit frame current hemisphere is dominated by the fragments of the struck quark alone; the 'spectator' proton remnants go entirely into the 'target' hemisphere, with much higher momentum. There is excellent acceptance for the current hemisphere in the central H1 detector. For example, even in the highest $Q^{2}$ interval of this analysis there is only an $\sim 8 \%$ contribution of tracks from the Forward Track Detector. The HCM current hemisphere, in contrast, has an energy scale of $W / 2$ rather than $Q / 2$ and generally can not be seen in its entirety. This makes comparisons with a complete $e^{+} e^{-}$interaction hemisphere somewhat easier in the Breit frame.

When QCD corrections to the parton model are considered, the incident parton carries, in general, a proportion of the incident proton's momentum that is larger than $x$ and has an energy larger than $Q / 2$. Furthermore, since the four-momentum of the photon is fixed the energy seen in the current hemisphere may be either greater than or less than $Q / 2$. In an $e^{+} e^{-}$interaction it is also the case that the energy in any given hemisphere may be greater than or less than $E^{*} / 2$. The effects of final state radiation in $e p$ DIS and $e^{+} e^{-}$ interactions are similar, but other LO QCD processes which affect DIS, initial state QCD radiation and boson gluon fusion (BGF), have no equivalent in $e^{+} e^{-}$annihilation. Note that, to order $\alpha_{s}$, i.e. two exiting partons as well as the spectator system, DIS events may have no energy in the current hemisphere when the incident parton has energy above $Q$, other than that due to hadronisation [3]. Momentum conservation ensures that this situation never exists in any given hemisphere of an $e^{+} e^{-}$interaction.

\section{Evolution of the Fragmentation Function}

The $e p$ Breit frame equivalent of the $e^{+} e^{-}$scaled hadron momentum $x_{p}=2 p_{\text {hadron }} / E^{*}$ is $x_{p}=2 p_{\text {hadron }} / Q$, where only hadrons in the current hemisphere are considered. The event normalised distribution $D\left(x_{p}, Q^{2}\right)=\left(1 / N_{\text {evts }}\right) \times d n_{\text {tracks }}^{ \pm} / d x_{p}$, the fragmentation function, characterises the processes by which partons shower and then hadronise. In this paper, the intention is to present the spectra of charged particles originating from the primary vertex following the fragmentation of light quarks.

At high enough energies and for light enough final state particles, fragmentation functions approximately scale and are "soft", rising rapidly at small $x_{p}$ and peaking near $x_{p}=0$. As the energy of the initial parton increases, $D\left(x_{p}, Q^{2}\right)$ evolves into an even softer function with increased probability for low $x_{p}$ hadrons at the expense of high $x_{p}$. This scaling violation can be seen with the low and high $Q^{2}$ data as a function of $x_{p}$ in Fig. 1. (a) or in Fig. 2 where the fragmentation function is plotted as a function of $Q$ for different intervals of $x_{p}$. Typically, in this analysis, the intervals of $x_{p}$ are much greater than the resolution $(\sim 6 \% \mathrm{rms})$. In these figures the $\mathrm{H} 1$ data are compared with equivalent data from $e^{+} e^{-}$experiments [18] plotted as a function of $E^{*}$. Most published $e^{+} e^{-}$results refer to full event multiplicities. Here, as elsewhere in this analysis, track multiplicity data from $e^{+} e^{-}$experiments have been halved to correspond to the fragmentation of one timelike quark. Note that $\mathrm{H} 1$ as a single experiment is able to measure the violation for a wide range of $Q$. Fig. 2 also displays a prediction from a leading order QCD plus hadronisation Monte Carlo (DJANGO6 [12]) calculation. The scaling violation effect has a similar origin to the scaling violations in structure functions, and with next to leading 
order calculations may be used as a test of perturbative QCD [19].
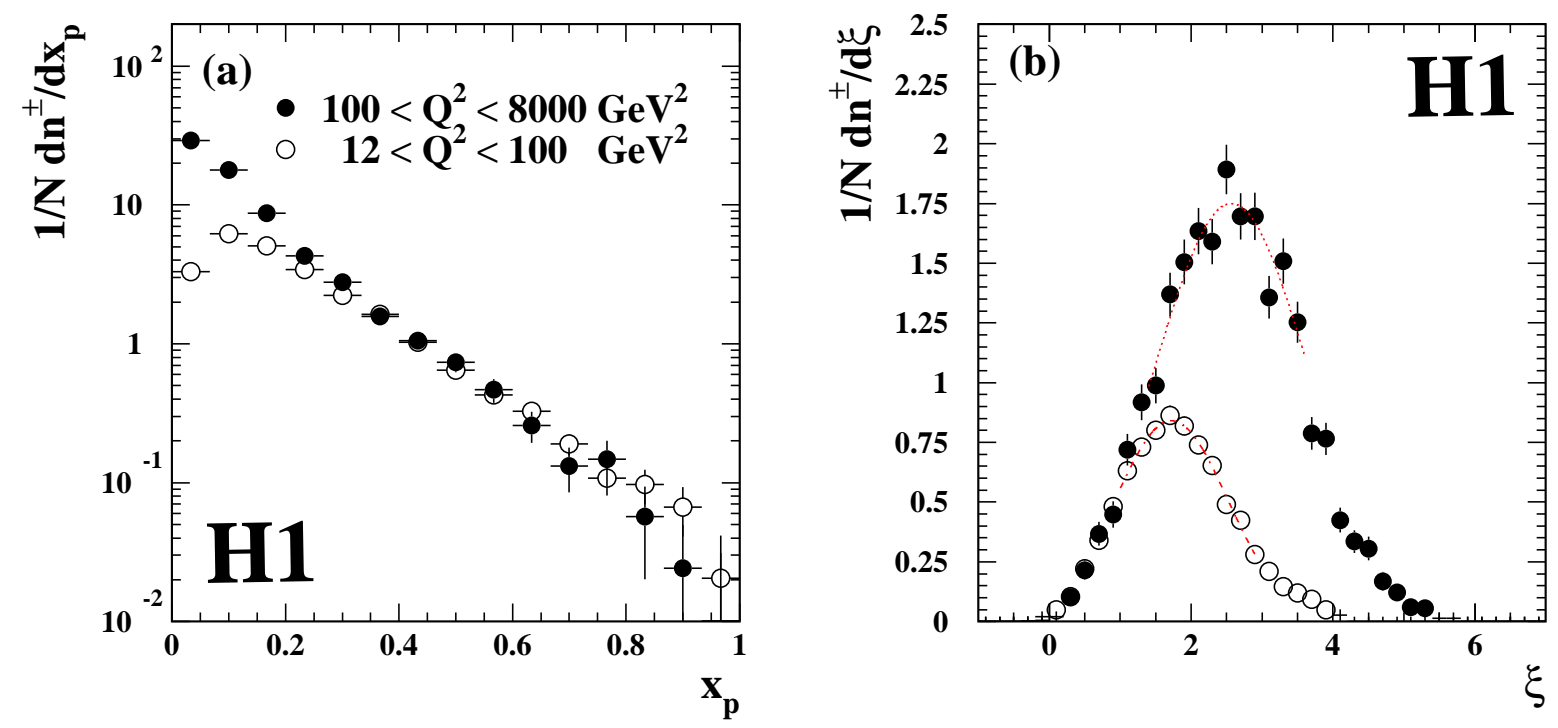

Figure 1: The current hemisphere fragmentation function as a function of (a) $x_{p}$ and (b) $\xi$ shown separately for the low (open circles) and high (closed circles) $Q^{2}$ data samples. Statistical and systematic errors are added in quadrature. The dashed and dotted lines show the results of Gaussian fits.

When the fragmentation function is plotted as a function of the variable $\xi=\ln \left(1 / x_{p}\right)$ the turn-over region is expanded. According to the expectations of the Modified Leading Logarithmic Approximation (MLLA) and Local Parton Hadron Duality (LPHD), $D(\xi)$ is Gaussian in the neighbourhood of the peak [20]. Even the high statistics of this, compared with our earlier, analysis see no significant deviation from this Gaussian behaviour, as is clear in Fig. 1(b). Most track measurement problems occur with particles of very high or very low momenta and these are correlated with low and high $\xi$ respectively. To avoid any dependence on these areas the fits of this analysis are restricted to a region within one unit of the dimensionless $\xi$ on either side of the mean, but tests in which this range is varied by $\pm 20 \%$ show no systematic difference.

The evolution of the fragmentation function may be summarised by the $Q$, or $E^{*}$, dependence of the peak and width (dispersion) values of the fitted Gaussian as is shown in Fig. 3 and in Table 1. It is notable that the systematic effects discussed in section 3 contribute only a total $2 \%$ uncertainty to the peak value and have no significant effect on the width measurement. The results are compatible with those published earlier, which had considerably lower precision, as well as with those of an analysis [4] by the ZEUS collaboration. The results are also compatible with various $e^{+} e^{-}$experiments [18, 21, 22], where the relevant evolution variable is the centre of mass energy, $E^{*}$. For this comparison and for the consideration of multiplicities in the next section, these published $e^{+} e^{-}$data have been re-fitted by us to be in a directly comparable form. As well as the previously 


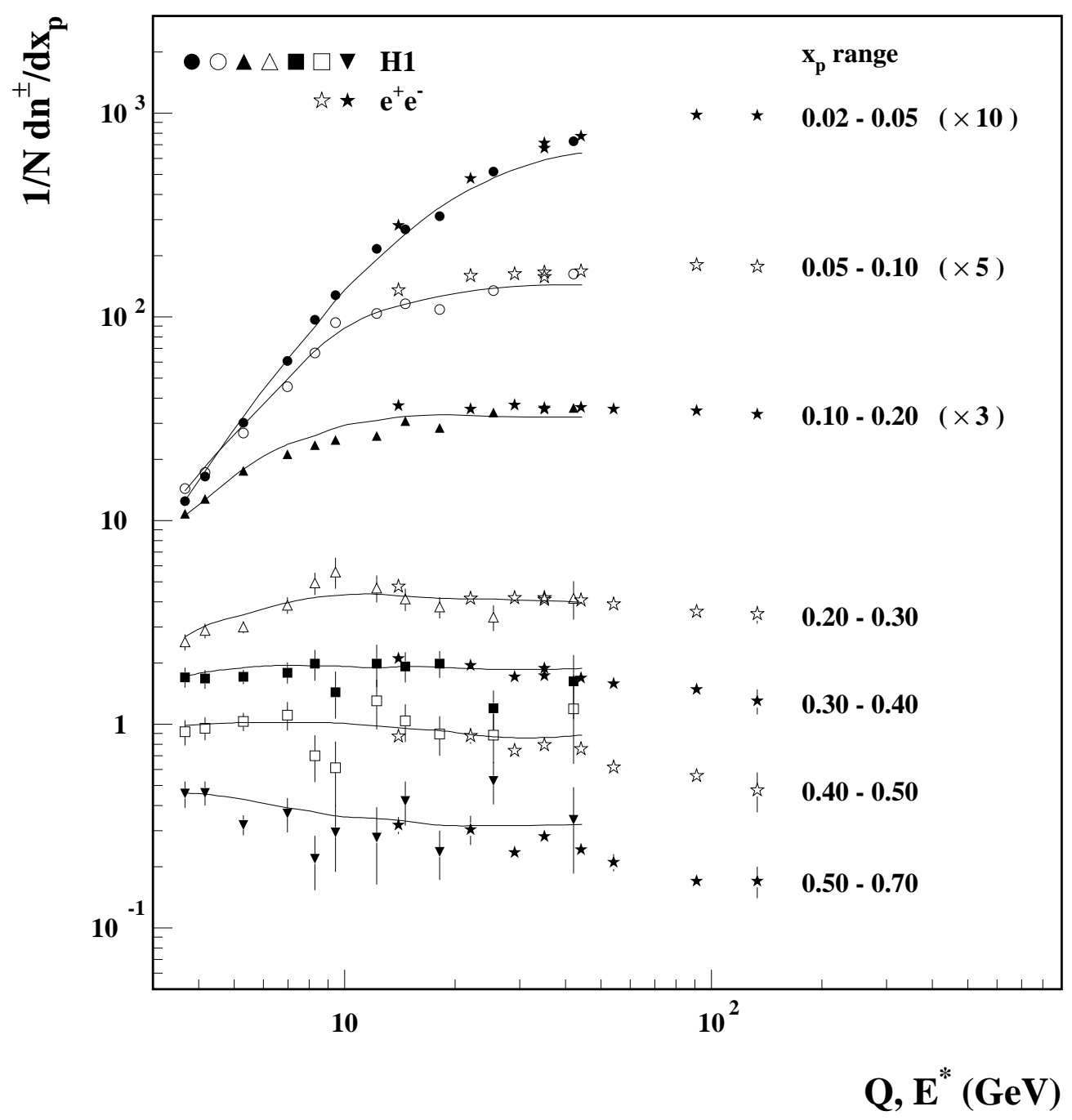

Figure 2: Scaling violations of the fragmentation function as a function of $E^{*}$ for $e^{+} e^{-}$ results (starred symbols), and as a function of $Q$ for $\mathrm{H} 1$ results (all other symbols) each within the same indicated intervals of the scaled momentum, $x_{p}$. Note that the data for the three lowest intervals of $x_{p}$ are multiplied by factors of 10, 5 and 3 respectively for clarity. Statistical and systematic errors are added in quadrature. The solid lines show the prediction of a DJANGO Monte Carlo calculation.

mentioned factor of two to convert $e^{+} e^{-}$event multiplicities to quark multiplicities, we also reduce published total multiplicity results by $8 \%$ to account for $K^{0}$ and $\Lambda$ decay tracks [23, 24], and by a variable factor below $3 \%$ to correct for the increased multiplicity from $b$ quark fragmentation [25] in $e^{+} e^{-}$.

Assuming gluon coherence MLLA/LPHD predicts a dependence of the evolution of the peak and width of the distribution on the dimensionless variable $Y=\ln \left(Q / \Lambda_{\text {eff }}\right)$, 

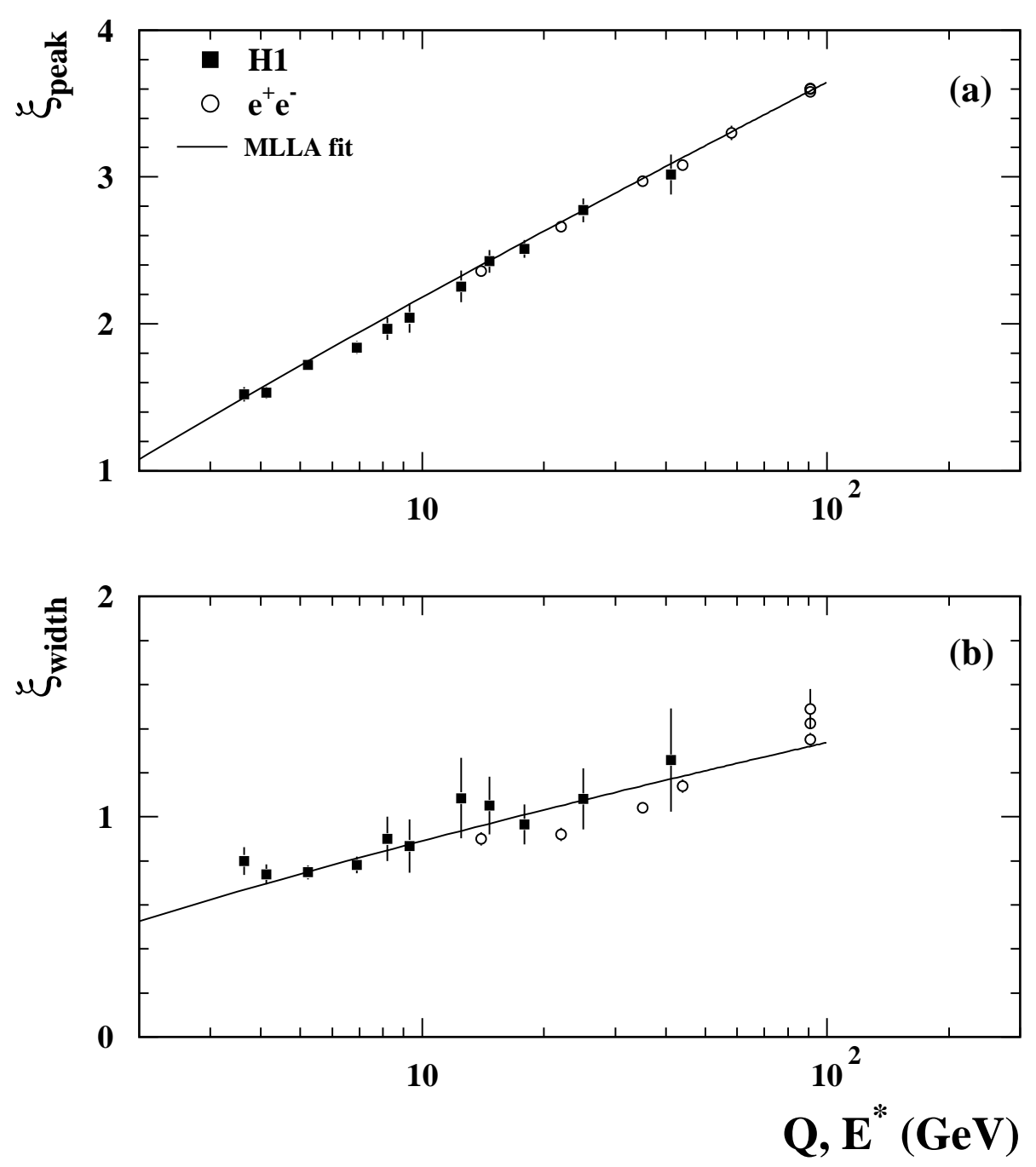

Figure 3: $H 1$ results (solid symbols, with statistical and systematic errors combined in quadrature) showing the evolution of (a) the peak and (b) the width of the fragmentation function as a function of $Q$ compared with $e^{+} e^{-}$results (open symbols) as a function of the centre of mass energy, $E^{*}$. The solid line is a fit to MLLA/LPHD expectations.

where $\Lambda_{\text {eff }}$ is a scale parameter determining a presumed cut-off of a parton shower. The prediction [20] gives the peak, $\xi_{\text {peak }}$ and the width, $\xi_{\text {width }}$, of the Gaussian approximation to be

$$
\begin{gathered}
\xi_{\text {peak }}=0.5 Y+c_{2} \sqrt{Y}+\mathcal{K} \\
\xi_{\text {width }}=\sqrt{Y^{\frac{3}{2}} / 2 c_{1}},
\end{gathered}
$$

where $c_{1}=\sqrt{36 N_{c} / b}$ and $c_{2}=B \sqrt{\frac{1}{16} b / N_{c}}$ with $b=\frac{11}{3} N_{c}-\frac{2}{3} N_{f}$ and $B=\left(\frac{11}{3} N_{c}+\right.$ $\left.\frac{2}{3} N_{f} / N_{c}^{2}\right) / b$ are constants dependent only on the number of contributing colours, $N_{c}$, and flavours, $N_{f}$ within the parton shower. For ease of comparison, we follow [21, 22] in the 


\begin{tabular}{|c||c|c|c||c|c|c|}
\hline \multicolumn{1}{|c||}{$\begin{array}{c}\langle Q\rangle \\
(\mathrm{GeV})\end{array}$} & \multicolumn{2}{c||}{ Total Current Hemisphere } & \multicolumn{3}{c|}{ Energy Flow Selected } \\
\cline { 2 - 7 } & $\langle n\rangle$ & Peak & Width & $\langle n\rangle$ & Peak & Width \\
\hline \hline 3.63 & $1.18 \pm 0.07$ & $1.52 \pm 0.05$ & $0.80 \pm 0.06$ & $1.77 \pm 0.11$ & $1.51 \pm 0.05$ & $0.76 \pm 0.07$ \\
\hline \hline 4.12 & $1.38 \pm 0.08$ & $1.53 \pm 0.04$ & $0.74 \pm 0.05$ & $1.85 \pm 0.11$ & $1.51 \pm 0.03$ & $0.68 \pm 0.04$ \\
\hline \hline 5.23 & $1.62 \pm 0.08$ & $1.72 \pm 0.03$ & $0.75 \pm 0.03$ & $2.13 \pm 0.11$ & $1.70 \pm 0.03$ & $0.76 \pm 0.03$ \\
\hline \hline 6.90 & $2.19 \pm 0.12$ & $1.84 \pm 0.04$ & $0.78 \pm 0.04$ & $2.60 \pm 0.15$ & $1.83 \pm 0.04$ & $0.77 \pm 0.05$ \\
\hline \hline 8.20 & $2.59 \pm 0.16$ & $1.97 \pm 0.08$ & $0.90 \pm 0.10$ & $3.10 \pm 0.20$ & $1.96 \pm 0.07$ & $0.87 \pm 0.10$ \\
\hline \hline 9.32 & $3.16 \pm 0.25$ & $2.04 \pm 0.10$ & $0.87 \pm 0.12$ & $3.49 \pm 0.29$ & $2.06 \pm 0.09$ & $0.84 \pm 0.11$ \\
\hline \hline 12.5 & $3.65 \pm 0.32$ & $2.26 \pm 0.11$ & $1.09 \pm 0.18$ & $3.93 \pm 0.35$ & $2.23 \pm 0.10$ & $1.03 \pm 0.17$ \\
\hline \hline 14.6 & $4.13 \pm 0.34$ & $2.43 \pm 0.08$ & $1.05 \pm 0.13$ & $4.45 \pm 0.30$ & $2.41 \pm 0.07$ & $1.04 \pm 0.13$ \\
\hline \hline 17.9 & $4.05 \pm 0.34$ & $2.51 \pm 0.06$ & $0.97 \pm 0.09$ & $4.28 \pm 0.36$ & $2.49 \pm 0.06$ & $0.98 \pm 0.10$ \\
\hline \hline 25.0 & $5.23 \pm 0.43$ & $2.77 \pm 0.08$ & $1.08 \pm 0.14$ & $5.28 \pm 0.44$ & $2.80 \pm 0.10$ & $1.18 \pm 0.18$ \\
\hline \hline 41.2 & $7.32 \pm 0.65$ & $3.02 \pm 0.14$ & $1.26 \pm 0.23$ & $7.19 \pm 0.65$ & $2.95 \pm 0.14$ & $1.19 \pm 0.20$ \\
\hline
\end{tabular}

Table 1: The average charged multiplicity, and the peak $\left(\xi_{\text {peak }}\right)$ and width $\left(\xi_{\text {width }}\right)$ of the fragmentation function as a function of $Q^{2}$, for the total current hemisphere of the Breit frame both with and without the energy flow selection discussed in section 6 . The errors are the sum of statistical and systematic uncertainties in quadrature.

assumption that this will be dominated by the light quarks and set $N_{f}=3$. The term $\mathcal{K}$ contains higher order corrections and is expected to be roughly constant and of order 1. The result of a simultaneous fit $\left(\chi^{2} / \mathrm{NDF}\right.$ of $\left.10 / 20\right)$ to the peak and width values obtained from the present $\mathrm{H} 1$ data alone is shown as a solid line in Fig. 3 and yields the values $\Lambda_{\text {eff }}=0.21 \pm 0.02 \mathrm{GeV}$ and $\mathcal{K}=-0.43 \pm 0.06$, in agreement with the values $\Lambda_{\text {eff }}=0.21 \pm 0.02 \mathrm{GeV}$ and $\mathcal{K}=-0.32 \pm 0.06$ obtained in an analysis [21] of the $\xi_{\text {peak }}$ evolution of combined $e^{+} e^{-}$data.

\section{The Average Charged Particle Multiplicity}

The area underneath the fragmentation function is the average charged multiplicity and this is given, after all corrections, in Table 1. It is shown as a function of $Q$ in Fig. 目, with ZEUS results [4] and with a curve which accurately parameterises [24] the average charged multiplicity from many $e^{+} e^{-}$experiments as a function of $E^{*}$. The error associated with this fit at any given energy is at the percent level.

There are predictions [26] that both the absolute average charged DIS multiplicity and the shape of the evolution with $Q^{2}$ should agree with that of $e^{+} e^{-}$experiments. As previous results indicated, although there was very good agreement between $\mathrm{H} 1$ and ZEUS [⿴囗⿴囗十 analyses and those of $e^{+} e^{-}$at high $Q\left(E^{*}\right)$, the DIS analyses give smaller average charged multiplicities at low values of $Q\left(E^{*}\right)$. The new data confirm that this is a large effect.

The discussion of section 4 indicated that the LO processes which are present in $e p$ 


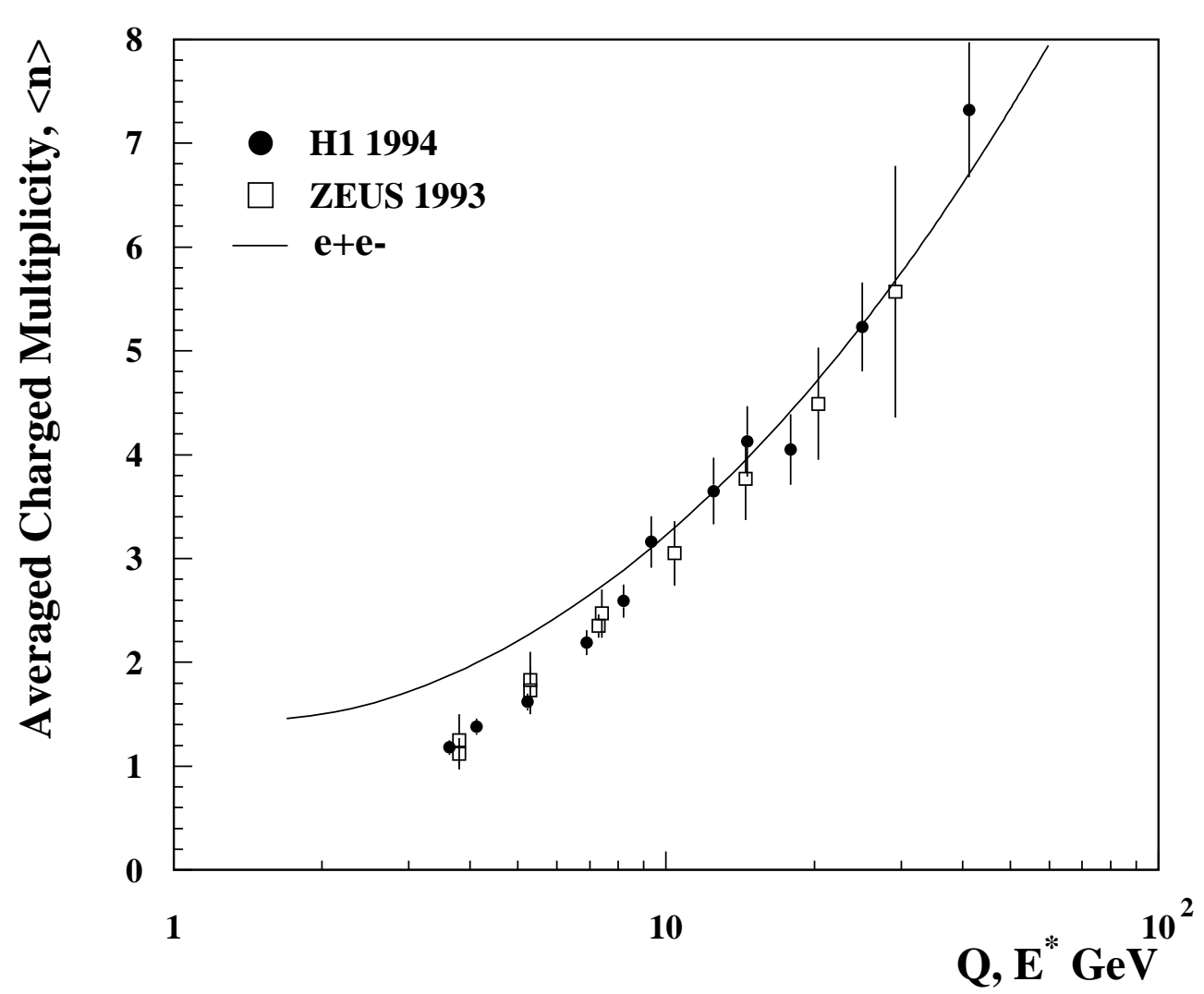

Figure 4: Average charged multiplicity in the current hemisphere of the Breit frame as a function of $Q$ for this analysis (solid circles) and for the ZEUS 1993 analysis (open squares). Statistical errors and systematic errors are added in quadrature. The curve is a fit to many $e^{+} e^{-}$results as a function of the centre of mass energy, $E^{*}$.

but not in $e^{+} e^{-}$interactions can produce a depletion of the current region of the Breit frame. Pure QPM interactions or events with only final state radiation (i.e. $e^{+} e^{-}$like events) might both be expected to have a current region hadronic state characteristic of a quark recoiling with energy $\sim Q / 2$ and with only a $z$ component of momentum. BGF events with a depleted or empty current hemisphere or events with significant initial state QCD radiation would, on the contrary, have a current region hadronic final state with significantly less energy at an angle to the $z$ axis. However, hadronisation effects might well mask these naïve expectations.

In order to investigate this experimentally, we add the four-momenta of all calorimeter energy clusters in the current region of the Breit frame. The energy component of the resultant four-momentum vector is defined to be $E_{z<0}$ and the angle it makes with the $z$ axis to be $\Theta_{B F}$. We then plot $E_{z<0} / Q$ against $\cos \Theta_{B F}$, as shown in Fig. 5(a,b) for the low and high $Q^{2}$ samples. From the above discussion we would expect that $e^{+} e^{-}$like events might cluster near $\left(\cos \Theta_{B F}=-1, E_{z<0} / Q=1 / 2\right)$ in this plot, whereas events from the other LO processes would either not appear in this plot or be spread away from this point, typically towards lower energies and larger angles in the current hemisphere, that is to the lower right-hand corner of this plot. The high $Q^{2}$ sample, where $\alpha_{s}$ is small, 
$x$ is large, and the proton has a much smaller gluon content, shows obvious clustering near the $(-1,0.5)$ point, while this is much less evident at low $Q^{2}$.

Monte Carlo simulations qualitatively confirm the expectations but it is hard to be quantitative, both because of the difficulty in strict definitions of LO QCD event classes and because experimental resolution has a large contribution to event migration on this plot. Nevertheless, it is possible [27] to investigate the effect of choosing ever more $e^{+} e^{-}$ like events by selecting according to the variable $R$, where $R^{2}=\left(E_{z<0} / Q-0.5\right)^{2}+$ $\left(\cos \Theta_{B F}+1\right)^{2}$. In order to compare with $e^{+} e^{-}$results, we divide $\langle n(R)\rangle$, the average multiplicity of events in an annulus of width $\Delta R=0.1$ at radius $R$, by the equivalent single-hemisphere $e^{+} e^{-}$multiplicity. There is a very small variation of $\langle Q\rangle$ with $R$, so this $e^{+} e^{-}$multiplicity is evaluated at an energy corresponding to the average value of $Q$ of the $\mathrm{H} 1$ data in that annulus. The behaviour of this ratio as a function of $R$ can be seen, with statistical error bars only, in Fig. 6(a,b). The systematic errors corresponding to the shaded region are as discussed in section 3. Events with a totally empty current hemisphere have zero multiplicity but no defined value of $R$, and are thus not included in these plots. As $R \rightarrow 0$ the average charged hadronic multiplicity increases, possibly even beyond the corresponding $e^{+} e^{-}$values, although it is not inconsistent with $e^{+} e^{-}$values given the systematic errors involved. Our Monte Carlo simulations also show this general behaviour.
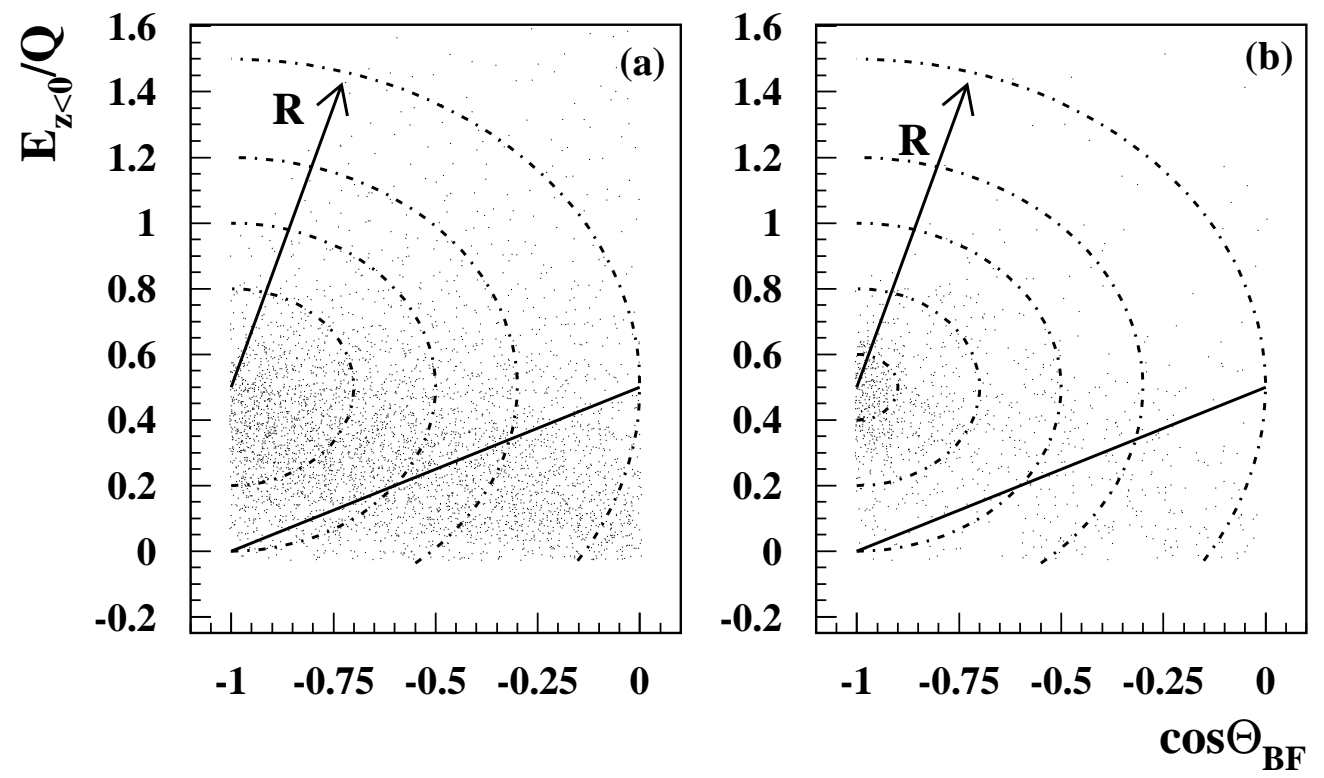

Figure 5: The total energy of the summed calorimeter cluster four-momentum vectors in the current hemisphere of the Breit frame is plotted as a fraction of the event $Q$ against the polar angle of the resultant vector, for (a) the low $Q^{2}$ and (b) the high $Q^{2}$ data sample. The annuli define the $R$ variable for Fig. 6 and the solid line indicates the Breit frame energy selection referred to in the text.

The above result and discussions motivate a cut to remove extreme low energy and off-axis events. This is shown as a solid line in Fig. 5(a,b) joining the points $(-1,0)$ and 

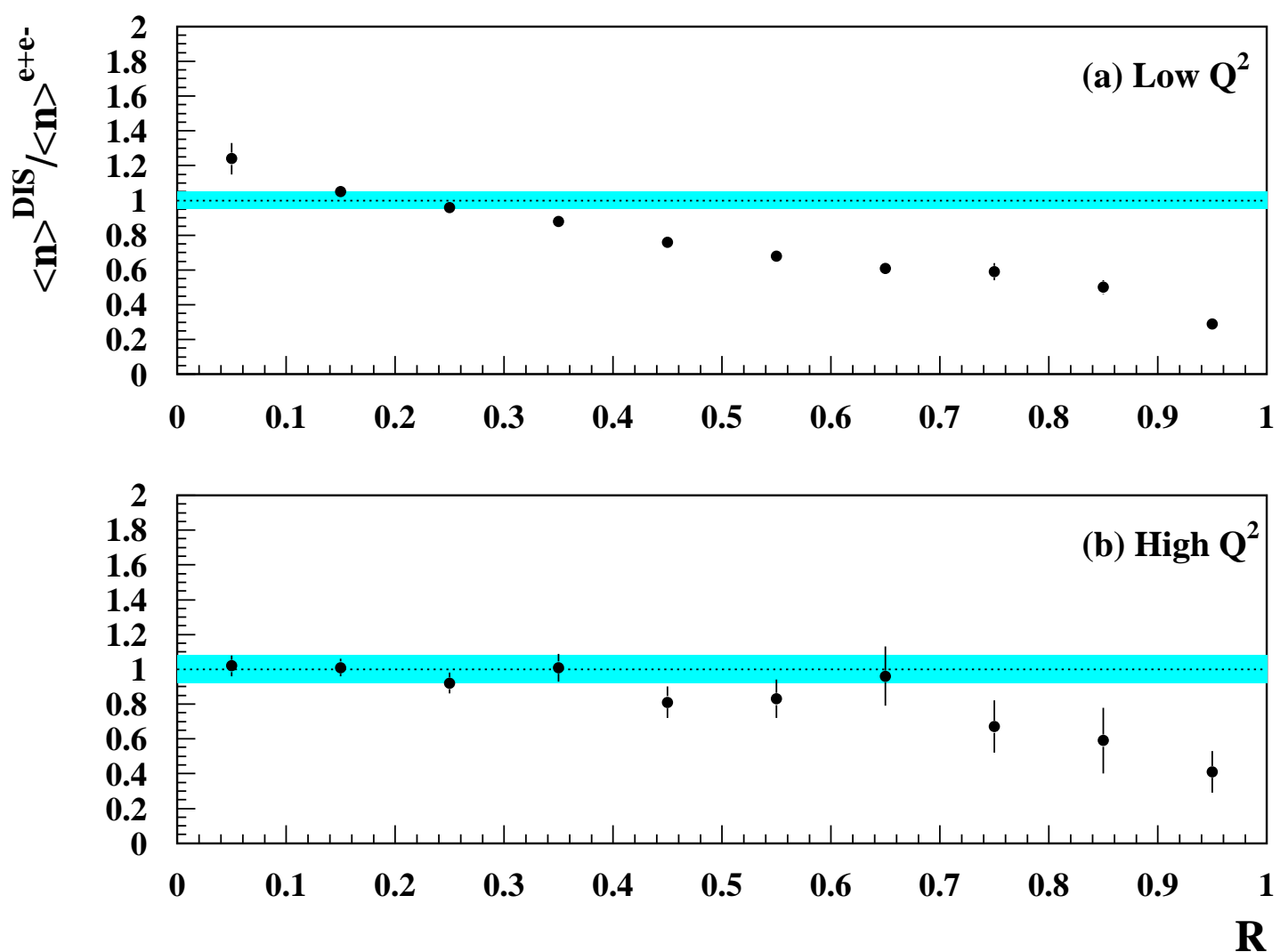

Figure 6: The H1 average charged multiplicity as a function of the radius defined in the text normalised to that of the single hemisphere expectation for $e^{+} e^{-}$events for (a) the low $Q^{2}$ and (b) the high $Q^{2}$ data sample. The H1 points have statistical error bars only. The shaded regions show the total systematic error.

$(0,0.5)$ in this plane and is termed the 'Breit frame energy flow selection' in the rest of this paper. It produces samples of 11735 events and 1005 events at low and high $Q^{2}$ respectively, which are less affected by the low-order QCD processes peculiar to DIS. This selection is by no means as exact as the technique of letting $R \rightarrow 0$ but in circumstances where the data are already binned in other variables, e.g. energy spectra or multiplicity distributions, gives an indication of whether or not distributions move in the direction of closer similarity between this data and $e^{+} e^{-}$data.

As is clear from Table 1, the peak and width values of the fragmentation function are insensitive to this energy flow selection procedure. The average multiplicity, however, is sensitive and as expected increases with this selection, especially at low $Q^{2}$.

\section{Invariant Energy Spectra}

The evolution of the fragmentation function at low energies, or high values of $\xi$, may be better studied using the event-normalised "invariant" spectrum, $\left(1 / N_{\text {evts }}\right) \times E d n_{\text {tracks }}^{ \pm} / d^{3} p$. According to the predictions of perturbative QCD based on the MLLA and LPHD, the hadronic spectrum at low momentum should be nearly independent of the energy of the parent parton [28]. Here we follow [28] in taking $d^{3} p=4 \pi p_{\text {hadron }}^{2} d p_{\text {hadron }}$ and by 
computing the energy, $E$, of each track as if it had a mass $Q_{0}\left(=270 \mathrm{MeV} \approx \Lambda_{Q C D}\right)$ given by a presumed cut-off mass for the parton shower, i.e. $E=\sqrt{Q_{0}^{2}+p_{\text {hadron }}^{2}}$. The data are corrected for acceptance with the same bin-by-bin method described in section 3 . The correction function is both smooth and close to unity, having an average value of around 1.15. Although there is always a loss of track acceptance at low laboratory momentum the boost reduces this loss in the Breit frame. This means that ep experiments can get closer to the critical low energy limit without serious loss of acceptance, than can $e^{+} e^{-}$ experiments.

The results are shown in Fig. 7(a,b) where the indicated errors contain contributions from statistical and (negligible) momentum measurement effects as well as systematic effects that have already been discussed and which grow slightly with track energy. The solid lines show MLLA/LPHD predictions at the relevant $Q$ values for a simple quark behaviour using the calculations of [28] which the authors show are in agreement with $e^{+} e^{-}$data. It is clear that at low $Q^{2}$ these predictions for the behaviour of quark fragments are in disagreement with the data but are significantly closer to data subject to the Breit frame energy flow selection described in the previous section. This selection makes little difference at high $Q^{2}$, where agreement is better anyway. The QCD predictions are very sensitive to the 'running' of the strong coupling constant, $\alpha_{s}$, as the energy scale alters in the parton shower. A prediction is shown for the same average energy, normalised to interpolated $\left\langle n^{ \pm}\right\rangle$multiplicities in $e^{+} e^{-}$experiments, but utilising a fixed value of $\alpha_{s}$ (set to 0.215 , a value which roughly describes the energy dependence of $\left\langle n^{ \pm}\right\rangle$and the slope of the first moment of the energy spectrum in $e^{+} e^{-}$experiments [29]). The data show that such an assumption is clearly untenable. This calculation is only in leading order so this cannot yet be turned around to compute a meaningful value of $\Lambda_{Q C D}$. Data subject to the energy flow selection are re-plotted in six intervals of $Q^{2}$ in Fig. U(c) in order to display the clear evidence for a common limit at low particle energy independent of event momentum transfer.

\section{Charged Particle Multiplicity Distributions}

Charged hadron multiplicity distributions are considerably more difficult to measure than average values. In this analysis we have tested a number of ways of extracting true distributions from observed distributions. The best method is found to be a simple bin-bybin correction procedure on the multiplicity distribution. This has, however, the problem of not modelling correlations which then gives the method a dependence on the Monte Carlo generator.

We tried two further methods to solve this difficulty. It is straightforward to use a Monte Carlo simulation to form a generated, or 'true', to reconstructed distribution transfer matrix, which is physics-generator independent. Inversion of this matrix in order to extract a true distribution from a raw input distribution has many problems due to the small number of high multiplicity events, resulting in the matrix becoming singular and unstable. We have also tried a method [17, 30] of approximating the inverted matrix with its inverse and then re-weighting the input Monte Carlo distribution in successive approximation iterations to minimise dependence on the initial generated distribution. 


\section{H1}
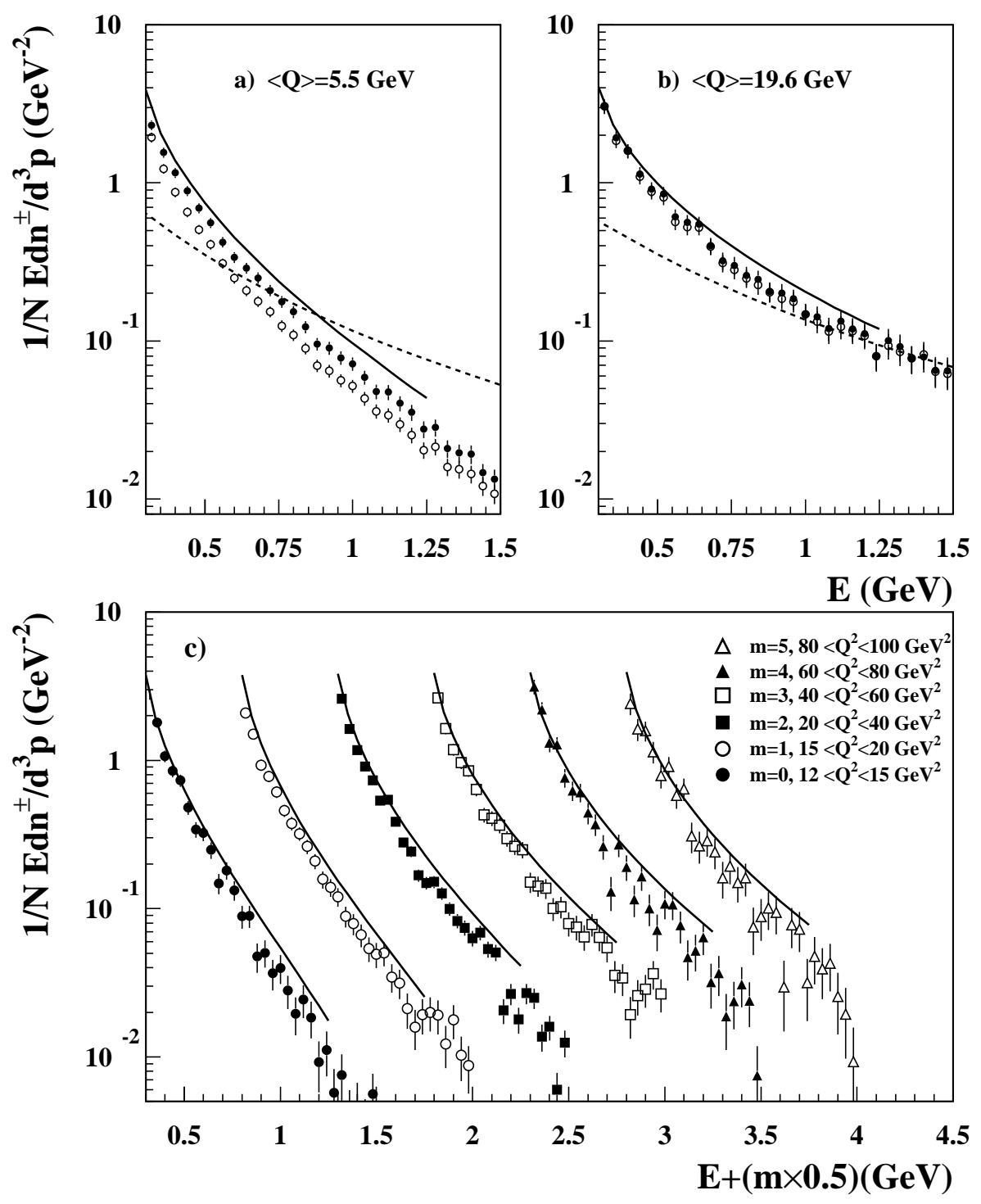

Figure 7: The invariant charged hadron energy spectrum in the current hemisphere (a) at low $Q^{2}$ and (b) at high $Q^{2}$. The data for all events are shown as open circles and those utilising the Breit frame energy flow selection as solid circles. The solid line is the prediction of MLLA/LPHD and the dashed line is the corresponding expectation for a non-running coupling constant, $\alpha_{s}$. In (c) the low $Q^{2}$ data are subjected to the energy flow selection but subdivided into the six indicated intervals of $Q^{2}$ and plotted with a regular incremental spacing of $0.5 \mathrm{GeV}$ on the abscissa. The solid line MLLA/LPHD expectation is calculated at the $\langle Q\rangle$ for each distribution. The error bars show the sum of statistical and systematic errors added in quadrature. 
The systematic errors of all three techniques have been estimated by degrading the output distributions back to the 'raw' (reconstructed) data level using the reliable 'generated to reconstructed' matrix. This can then be compared with the original, real input distribution. We find that the larger dependence on the generated distribution of the binby-bin correction method is still offset by overall smaller systematic errors and the ability to get meaningful corrections at high multiplicity when the statistics are low. We thus choose this method to present our results. Quantitatively, the average correction made per bin is $16 \%(32 \%)$ at low (high) $Q^{2}$ with an error of method assessed as $10 \%(17 \%)$.

The resulting multiplicity distributions are given in Table 2 and shown in Fig. 8 in the form of $P(n)$, the probability of observing a charged multiplicity $n$. These results are for the multiplicity distribution in the current hemisphere of all events and do not include any energy flow selection. The bins of $x$ and $Q^{2}$ are exclusive but, because of the event selections, are not totally occupied at the edges. This leads to only minor discrepancies in the average values of $x$ and $Q^{2}$ between neighbouring bins. In general the observation is that the results are compatible with a gradual shift to higher multiplicities with increasing $Q^{2}$ but with only a small change as a function of $x$ which might be due to minor bin edge effects. It is interesting to note the many zero-charged multiplicity events, especially at low $Q^{2}$ where we might expect higher-order QCD processes to dominate.

Integrating over $x$ gives smaller statistical errors and a greater reach in multiplicity. The evolution with $Q$ of the shape of the multiplicity distribution may be studied by normalising with the average charged hadron multiplicity to give the KNO [31] form $\Psi(z)=\langle n\rangle \times P(n)$, where $z=n /\langle n\rangle$. Equivalent single-hemisphere $e^{+} e^{-}$distributions [24, 32, 33] exhibit clear scaling in this variable but only for high $\left(E^{*}>20 \mathrm{GeV}\right)$ energies. In an analysis [17] of multiplicity distributions in a limited region of the hadronic centre of mass frame, the H1 collaboration has also observed good agreement with a common distribution as $W$ increases between 80 and $220 \mathrm{GeV}$. The results of this analysis in the Breit frame, shown in Fig. 9(a), are for $Q$ values much lower than the $W$ values and show a clear violation of KNO scaling, as also reported by the ZEUS collaboration [4]. For reference, we display two sets of lines joining $e^{+} e^{-}$data at an energy close to our largest $Q$ range [32 and also at LEP energies [24]. As with $e^{+} e^{-}$data, the H1 low $Q^{2}$ distribution starts with a flatter curve and a large contribution from low multiplicities. Then, as $Q^{2}$ increases, the data get closer to the high energy scaling $e^{+} e^{-}$distributions.

In Fig. 9(b) the distribution for low $Q^{2}$ events is compared with results from the $e^{+} e^{-}$ JETSET [34 parton shower model, using parameter settings determined by the DELPHI collaboration [35] at an energy set to be close to the mean value of the low $Q^{2}$ data. The data distribution is again much flatter but a better agreement is achieved when using the Breit frame energy flow selection discussed in section 6. This KNO scaling violation is also seen in ep DIS Monte Carlo generated events, where it is to a large extent caused by leading order perturbative QCD events, especially BGF events, which, as has already been noted, have significantly lower multiplicities in the current hemisphere of the Breit frame. It thus appears that $e p$ and $e^{+} e^{-}$data share a flattening of the KNO distribution at low energies but that there is an additional sensitivity to the effects of higher order QCD processes in the Breit frame which do not contribute to $e^{+} e^{-}$events. 

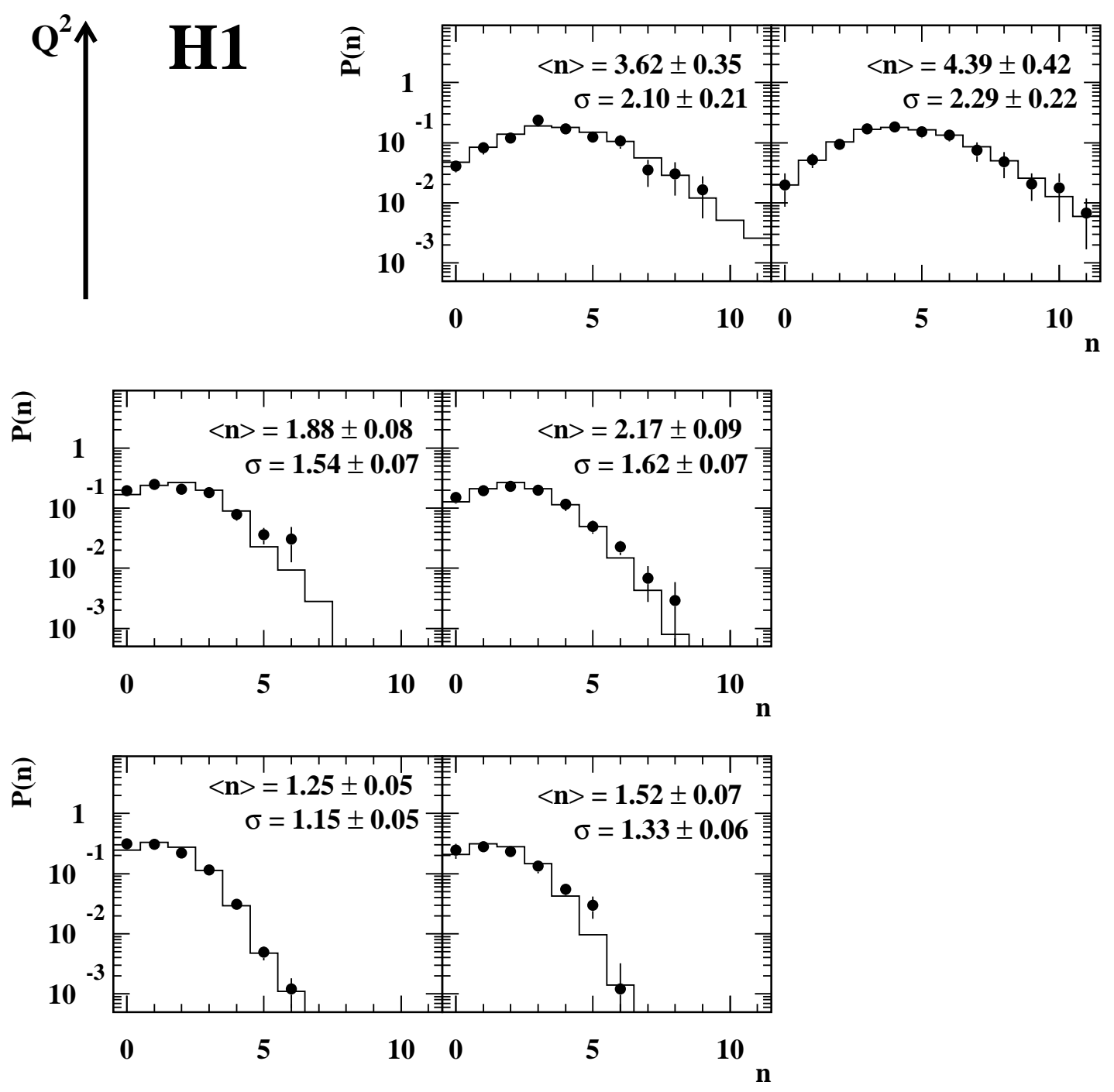

$\mathbf{X}$

Figure 8: The event-normalised charged hadron multiplicity distribution in the current region of the Breit frame, with statistical and systematic errors added in quadrature. Moving from bottom to top the distributions are for $12<Q^{2}<30 \mathrm{GeV}^{2}, 30<Q^{2}<80$ $\mathrm{GeV}^{2}$ and $100<Q^{2}<500 \mathrm{GeV}^{2}$, and from left to right the ranges in Bjorken-x are $6 \cdot 10^{-4}<x<2 \cdot 10^{-3}, 2 \cdot 10^{-3}<x<1 \cdot 10^{-2}$ and $1 \cdot 10^{-2}<x<2 \cdot 10^{-1}$. The average

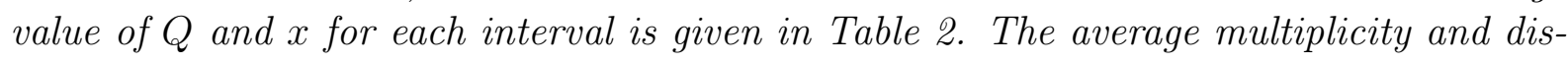
persion are shown at the top of each distribution. The histograms show the prediction of a DJANGO Monte Carlo calculation. 


\begin{tabular}{|c|c|c|c|c|c|}
\hline \multirow{3}{*}{$\begin{array}{c}\langle Q\rangle \\
(\mathrm{GeV})\end{array}$} & \multirow{3}{*}{$\begin{array}{l}<x> \\
\left(\times 10^{3}\right)\end{array}$} & $P(0)$ & $P(1)$ & $P(2)$ & $P(3)$ \\
\hline & & $P(4)$ & $P(5)$ & $P(6)$ & $P(7)$ \\
\hline & & $P(8)$ & $P(9)$ & $P(10)$ & $P(11)$ \\
\hline \multirow[t]{3}{*}{4.15} & \multirow[t]{3}{*}{1.26} & $\bar{~} 0.312 \pm 0.025$ & 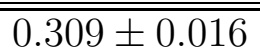 & 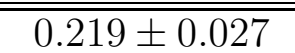 & 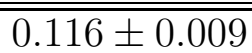 \\
\hline & & $0.031 \pm 0.005$ & $0.005 \pm 0.001$ & $0.0012 \pm 0.0006$ & - \\
\hline & & - & - & - & - \\
\hline \multirow[t]{3}{*}{4.53} & \multirow[t]{3}{*}{2.55} & $0.246 \pm 0.070$ & $0.281 \pm 0.025$ & $0.233 \pm 0.042$ & $0.133 \pm 0.031$ \\
\hline & & $0.055 \pm 0.011$ & $0.030 \pm 0.012$ & $0.0012 \pm 0.0020$ & - \\
\hline & & - & - & - & - \\
\hline \multirow[t]{3}{*}{6.20} & \multirow[t]{3}{*}{1.58} & $0.194 \pm 0.023$ & $0.250 \pm 0.026$ & $0.205 \pm 0.025$ & $0.182 \pm 0.023$ \\
\hline & & $0.079 \pm 0.017$ & $0.036 \pm 0.011$ & $0.031 \pm 0.018$ & - \\
\hline & & - & - & - & - \\
\hline \multirow[t]{3}{*}{7.04} & \multirow[t]{3}{*}{4.10} & $0.150 \pm 0.031$ & $0.195 \pm 0.013$ & $0.229 \pm 0.025$ & $0.199 \pm 0.020$ \\
\hline & & $0.116 \pm 0.026$ & $0.049 \pm 0.012$ & $0.023 \pm 0.006$ & $0.007 \pm 0.004$ \\
\hline & & $0.003 \pm 0.003$ & - & - & - \\
\hline \multirow[t]{3}{*}{14.5} & \multirow[t]{3}{*}{6.82} & $0.041 \pm 0.009$ & $0.083 \pm 0.019$ & $0.119 \pm 0.024$ & $\overline{0.236 \pm 0.049}$ \\
\hline & & $0.169 \pm 0.024$ & $0.124 \pm 0.024$ & $0.106 \pm 0.027$ & $0.035 \pm 0.017$ \\
\hline & & $0.030 \pm 0.017$ & $0.017 \pm 0.011$ & - & - \\
\hline \multirow[t]{3}{*}{17.3} & \multirow[t]{3}{*}{2.34} & $0.020 \pm 0.011$ & $0.052 \pm 0.014$ & $0.093 \pm 0.018$ & $0.171 \pm 0.031$ \\
\hline & & $0.184 \pm 0.026$ & $0.151 \pm 0.032$ & $0.135 \pm 0.029$ & $0.075 \pm 0.026$ \\
\hline & & $0.048 \pm 0.022$ & $0.021 \pm 0.010$ & $0.018 \pm 0.013$ & $0.007 \pm 0.005$ \\
\hline
\end{tabular}

Table 2: The probability per event, $P(n)$, of observing a charged hadronic multiplicity of $n$ in the current hemisphere of the Breit frame as a function of $Q$ and $x$. The errors are the sum of statistical and systematic errors in quadrature. 

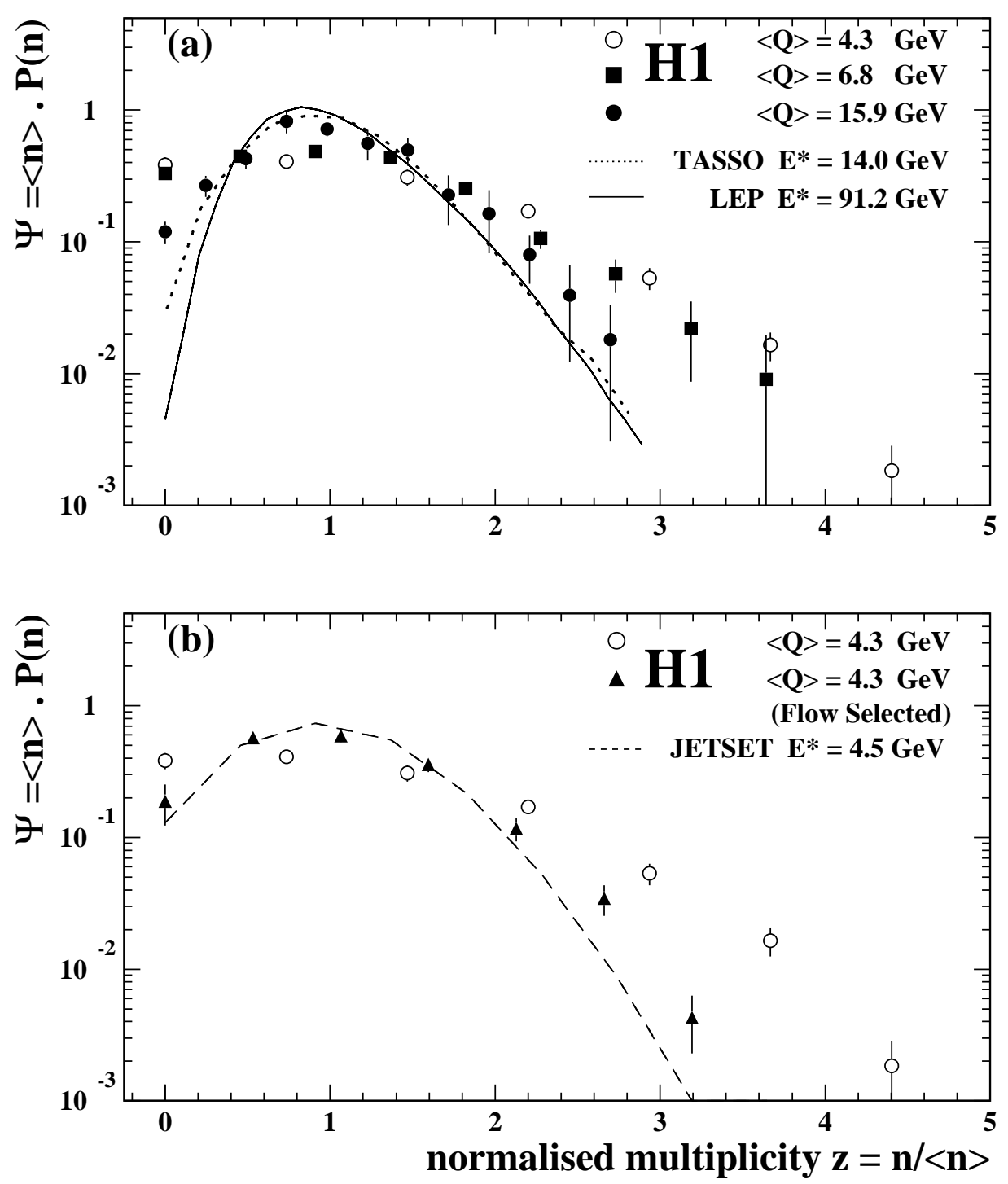

Figure 9: (a) The charged hadronic multiplicity distribution for $H 1$ data expressed in terms of the KNO variables for $12<Q^{2}<30 \mathrm{GeV}^{2}$ (open circles), $30<Q^{2}<80 \mathrm{GeV}^{2}$ (squares) and $100<Q^{2}<500 \mathrm{GeV}^{2}$ (solid circles). (b) A repeat of the $12<Q^{2}<30 \mathrm{GeV}^{2} \mathrm{H1}$ data with (solid triangles) and without (open circles) the Breit frame energy flow selection. Statistical and point to point systematic error bars are added in quadrature in both figures. The dotted and solid lines represent the single hemisphere KNO functions for $e^{+} e^{-}$data at $E^{*}=14$ and $91 \mathrm{GeV}$ respectively and the dashed line shows the result from the $e^{+} e^{-}$ JETSET Monte Carlo at an $E^{*}$ value close to the $Q$ value of the ep data. 


\section{Summary}

The Breit frame fragmentation function analysis has been repeated with the higher statistics of 1994 data and is now extended to give multiplicity distributions as functions of both $x$ and $Q^{2}$. Invariant energy spectra are shown to be incompatible with a calculation utilising a constant value for $\alpha_{s}$. We discuss a method of discriminating against events in kinematic regions dominated by QCD processes which do not contribute to $e^{+} e^{-}$interactions. We show that some discrepancies between ep Breit frame current hemisphere and $e^{+} e^{-}$average charged multiplicities, including an observed violation of KNO scaling at low $Q^{2}$, decrease as conditions are progressively made more comparable. With a selection which gives similar kinematic conditions to that of a single hemisphere $e^{+} e^{-}$annihilation, the ep energy spectra can be shown to be compatible with a common, $Q^{2}$-independent, low momentum limit. In summary, the data give strong support to the concept of quark fragmentation universality.

\section{Acknowledgments}

We are grateful to the HERA machine group whose outstanding efforts have made and continue to make this experiment possible. We thank the engineers and technicians for their work in constructing and now maintaining the $\mathrm{H} 1$ detector, our funding agencies for financial support, the DESY technical staff for continual assistance, and the DESY directorate for the hospitality which they extend to the non-DESY members of the collaboration. We are grateful to S. Lupia for kind assistance with obtaining MLLA/LPHD predictions.

\section{References}

[1] H1 Collaboration, S. Aid et al., Nucl. Phys. 445B (1995) 3.

[2] H.F. Jones, Nuovo Cimento 40A (1965) 1018.

[3] K.H. Streng et al., Z. Phys. C2 (1979) 237.

[4] ZEUS Collaboration, M. Derrick et al., Z. Phys. C67 (1995) 93.

[5] H1 Collaboration, I. Abt et al., DESY preprint 93-103 (1993), Nucl. Instr. Meth. A386 (1997) 310 (Vol 1) and ibid. p.348 (Vol 2).

[6] J. Bürger et al., Nucl. Instr. Meth. A279 (1989) 217.

[7] H1 FTD group, S. Burke et al., Nucl. Instr. Meth. A373 (1996) 227.

[8] H1 calorimeter group, B. Andrieu et al., Nucl. Instr. Meth. A336 (1993) 460.

[9] H1 BEMC group, J. Ban et al., Nucl. Instr. Meth. A372 (1996) 399. 
[10] H1 Collaboration, S. Aid et al., Nucl. Phys. 470B (1996) 3.

[11] Proceedings of the study of an ep facility for Europe, Ed. U. Amaldi, DESY 79/48 (1979) p391-4.

[12] G.A. Schuler and H. Spiesberger, Proceedings of the Workshop: Physics at HERA, vol. 3 eds. W. Buchmüller, G. Ingelman, DESY (1992) 1419. We use version 6.1 with QED radiation simulated with HERACLES 4.4 when correcting data. QCD radiation is simulated by the colour dipole model (ARIADNE 4.03/4.05) and hadronisation with the LUND string model in JETSET 7.4. We use MRSH parton density parametrizations at low $Q^{2}$ and GRV at high $Q^{2}$.

[13] R. Brun et al., GEANT3 User's guide, CERN-DD/EE 84-1, Geneva (1987).

[14] LEPTO 6.5 Generator with GRV parton density functions ; G. Ingelman, Proceedings of the Workshop: Physics at HERA, vol. 3 eds. W. Buchmüller, G. Ingelman, DESY (1991) 1366.

[15] HERWIG 5.8 Generator with MRSH parton density function, and no soft underlying event ; G. Marchesini et al., Computer Phys. Comm. 67 (1992) 32.

[16] P. Dixon, University of Lancaster thesis, in preparation.

[17] H1 Collaboration, S. Aid et al., Z. Phys. C72 (1996) 573.

[18] TASSO Collaboration, W. Braunschweig et al., Z. Phys. C47 (1990) 187, Phys. Lett. 311B (1993) 408; DELPHI Collaboration, P. Abreu et al., Phys. Lett. 311B (1993) 408; OPAL Collaboration, G. Alexander et al., Z. Phys. C72 (1996) 191.

[19] G.D. Cowan Glasgow XXVII HEP Conference 1994 proceedings p.883, IoP Publishing Ltd. 1995; J. Binnewies, B.A. Kniehl and G. Kramer Phys. Rev. 52D (1995) 4947 and Phys. Rev. 53D (1996) 3573.

[20] Yu.L. Dokshitzer, V.A. Khoze, A.H. Mueller and S.I. Troyan, "Basics of Perturbative QCD" Editions Frontières (1991); Ya.I. Azimov, Yu.L. Dokshitzer, V.A. Khoze, and S.I. Troyan, Z. Phys. C31 (1986) 213, Z. Phys. C27 (1985) 65; L.V. Gribov, Yu.L. Dokshitzer, V.A. Khoze, and S.I. Troyan, Phys. Lett. 202B (1988) 276.

[21] OPAL Collaboration, M.Z. Akrawy et al., Phys. Lett. 247B (1990) 617.

[22] ALEPH Collaboration, R. Barate et al., CERN PPE 96-186.

[23] TASSO Collaboration, M. Althoff et al., Z. Phys. C22 (1984) 307.

[24] OPAL Collaboration, P.D. Acton et al., Z. Phys. C53 (1992) 539.

[25] OPAL Collaboration, R. Akers et al., Z. Phys. C61 (1994) 209.

[26] A.Basetto, Nucl. Phys. 202B (1982) 493. 
[27] D. Kant, University of London thesis, also published by the Rutherford Appleton laboratory as RAL-TH-96-008.

[28] V.A. Khoze, S. Lupia, and W. Ochs, Phys. Lett. 394B (1997) 179.

[29] S. Lupia, private communication.

[30] P. Van Mechelen, University of Antwerp thesis, in preparation.

[31] Z. Koba, H.B. Nielsen and P. Olesen, Nucl. Phys. 40B (1972) 317.

[32] TASSO Collaboration, W. Braunschweig et al., Z. Phys. C45 (1989) 193.

[33] DELPHI Collaboration, P. Abreu et al. Z. Phys. C56 (1992) 63.

[34] T. Sjöstrand, Computer Phys. Comm. 39 (1986) 32; T. Sjöstrand, M. Bengtsson, Computer Phys. Comm. 43 (1987) 32.

[35] DELPHI Collaboration, P. Abreu et al. Z. Phys. C73 (1996) 11. 